\title{
Advances and challenges in interval-valued fuzzy logic
}

\author{
C. Cornelis*, G. Deschrijver, E.E. Kerre \\ Fuzziness and Uncertainty Modelling Research Unit, Department of Applied Mathematics and Computer Science, Ghent University, \\ Krijgslaan 281 (S9), 9000 Gent, Belgium
}

Available online 28 October 2005

\begin{abstract}
Among the various extensions to the common [0,1]-valued truth degrees of "traditional" fuzzy set theory, closed intervals of $[0,1]$ stand out as a particularly appealing and promising choice for representing imperfect information, nicely accommodating and combining the facets of vagueness and uncertainty without paying too much in terms of computational complexity. From a logical point of view, due to the failure of the omnipresent prelinearity condition, the underlying algebraic structure $\mathscr{L}^{I}$ falls outside the mainstream of the research on formal fuzzy logics (including MV-, BL- and MTL-algebras), and consequently so far has received only marginal attention. This comparative lack of interest for interval-valued fuzzy logic has been further strengthened, perhaps, by taking for granted that its algebraic operations amount to a twofold application of corresponding operations on the unit interval. Abandoning that simplifying assumption, however, we may find that $\mathscr{L}^{I}$ reveals itself as a very rich and noteworthy structure allowing the construction of complex and surprisingly well-behaved logical systems. Reviewing the main advances on the algebraic characterization of logical operations on $\mathscr{L}^{I}$, and relating these results to the familiar completeness questions (which remain as major challenges) for the associated formal fuzzy logics, this paper paves the way for a systematic study of interval-valued fuzzy logic in the narrow sense.
\end{abstract}

(C) 2005 Elsevier B.V. All rights reserved.

Keywords: Interval-valued fuzzy logic; Logical connectives; Algebraic structures; Representability; Fuzzy logics

\section{Introduction}

Interval-valued fuzzy set theory (apparently introduced independently in the mid-seventies by Grattan-Guinness [11], Jahn [14], Sambuc [19] and Zadeh [21]) is an increasingly popular extension of fuzzy set theory where traditional [0, 1]valued membership degrees are replaced by intervals in $[0,1]$ that approximate the (partially unknown) exact degrees. Hence, not only vagueness (lack of sharp class boundaries), but also a feature of uncertainty (lack of information) can be addressed intuitively. Moreover, interval-valued fuzzy sets (IVFSs) are considerably easier to handle in practice than the similarly inspired type-2 fuzzy sets (of which IVFSs are in fact a special case, called "interval type-2 fuzzy sets" in that context, see e.g. [17]); as further evidence of their wide relevance, they also subsume the syntactically equivalent frameworks of Atanassov's intuitionistic fuzzy sets [1] and of Gau and Buehrer's vague sets [7].

\footnotetext{
* Corresponding author.

E-mail addresses: Chris.Cornelis@UGent.be (C. Cornelis), Glad.Deschrijver@UGent.be (G. Deschrijver), Etienne.Kerre@UGent.be (E.E. Kerre)

URL: http://www.fuzzy.UGent.be (Fuzziness and Uncertainty Modelling).
} 
Their potential to approximate reasoning applications was noticed early on, for instance by Türkşen [20] and Gorzałczany [10], who experimented with the extension of logical operations to interval-valued truth degrees. Unlike the steep rise in interest that we have experienced over the past decade for t-norm-based (residuated) fuzzy logics defined on the unit interval (see e.g. [12,18,6]), a similar development did not take place for IVFSs, and a unified, formal treatment of interval-valued fuzzy logics is still lacking. As we will argue in the remainder of this paper, two important obstacles are responsible for this: an underestimation of the richness of the associated algebraic structure, and a marked preference within the research community for prelinear algebraic structures.

\section{The lattice $\mathcal{L}^{I}$}

From an algebraic perspective, an attractive and conceptually straightforward framework in which to develop the theory of IVFSs and their associated operations is provided by Goguen's $\mathcal{L}$-fuzzy sets [9]. Indeed, the structure $\mathcal{L}^{I}=$ $\left(L^{I}, \leqslant L^{I}\right)$ given by

$$
\begin{aligned}
& L^{I}=\left\{\left[x_{1}, x_{2}\right] \mid\left(x_{1}, x_{2}\right) \in[0,1]^{2} \text { and } x_{1} \leqslant x_{2}\right\}, \\
& {\left[x_{1}, x_{2}\right] \leqslant L_{L^{I}}\left[y_{1}, y_{2}\right] \Longleftrightarrow x_{1} \leqslant y_{1} \text { and } x_{2} \leqslant y_{2}}
\end{aligned}
$$

is a complete lattice, and hence an IVFS $A$ in $U$ can be seen as an $\mathcal{L}^{I}$-fuzzy set in $U$, i.e. as a mapping from $U$ to the ordered structure $\mathcal{L}^{I}$. By virtue of the ordering $\leqslant{ }_{L^{I}}$, definitions of graded logical connectives like negators, t-(co)norms and implicators are readily obtained on $\mathcal{L}^{I}$. For instance, a t-norm on $\mathcal{L}^{I}$ is a commutative, associative, $\leqslant_{L^{I} \text {-increasing }}$ $\left(L^{I}\right)^{2} \rightarrow L^{I}$ mapping $\mathcal{T}$ satisfying $\mathcal{T}([1,1], x)=x$, for all $x$ in $L^{I}$.

Note that the infimum $\wedge$ (meet) and supremum $\vee$ (join) on $\mathcal{L}^{I}$ are given by, for $\left[x_{1}, x_{2}\right],\left[y_{1}, y_{2}\right]$ in $L^{I}$,

$$
\begin{aligned}
& {\left[x_{1}, x_{2}\right] \wedge\left[y_{1}, y_{2}\right]=\left[\min \left(x_{1}, y_{1}\right), \min \left(x_{2}, y_{2}\right)\right],} \\
& {\left[x_{1}, x_{2}\right] \vee\left[y_{1}, y_{2}\right]=\left[\max \left(x_{1}, y_{1}\right), \max \left(x_{2}, y_{2}\right)\right] .}
\end{aligned}
$$

In other words, $\wedge$ and $\vee$ emerge as componentwise applications of their counterparts on $[0,1]$. For many authors (see e.g. $[8,15]$ ), this observation serves as adequate justification to restrict the definition of logical connectives to componentwise operations, which amounts to the implicit acknowledgment that the research on IVFSs would reduce to a mere twofold (and hence not very challenging) application of the results from fuzzy set theory. For instance, Jenei [15] defined a t-norm on $L^{I}$ as an $\left(L^{I}\right)^{2} \rightarrow L^{I}$ mapping $\mathcal{T}$ such that there exist t-norms $T_{1}, T_{2}$ on [0,1] for which $\mathcal{T}\left(\left[x_{1}, x_{2}\right],\left[y_{1}, y_{2}\right]\right)=\left[T_{1}\left(x_{1}, y_{1}\right), T_{2}\left(x_{2}, y_{2}\right)\right]$. Such an assumption however not only disregards the natural partial ordering $\leqslant_{L^{I}}$, it also remains blind to some of the most significant and exciting aspects of IVFS theory.

Definition 1 (Deschrijver et al [5]). Let $\mathcal{T}$ be a t-norm on $\mathcal{L}^{I}$. We say that $\mathcal{T}$ is t-representable if there exist t-norms $T_{1}$ and $T_{2}$ on $[0,1]$ such that, for all $x, y$ in $L^{I}, \mathcal{T}(x, y)=\left[T_{1}\left(x_{1}, y_{1}\right), T_{2}\left(x_{2}, y_{2}\right)\right]$.

Clearly, by this definition Jenei's t-norms are t-representable. In [5,3] it is shown on the other hand that not all t-norms on $\mathcal{L}^{I}$ are t-representable; moreover, as will be clear from the sequel, some of the non t-representable t-norms are far superior to their t-representable counterparts in terms of the algebraic properties that they satisfy.

In the following section, we will use the structure $\mathcal{L}^{I}$ as the algebraic basis for developing logical calculi in a natural and interpretation-independent manner.

\section{Algebraic structures for interval-valued fuzzy logics-a brief synopsis of the state of the art}

First, we recall some structures commonly used in fuzzy logic (see e.g. $[12,18,6,2])$.

Definition 2. An algebraic structure $\mathcal{L}=(L, \wedge, \vee, *, \Rightarrow, 0,1)$ is called a residuated lattice if

(RL.1) $(L, \wedge, \vee)$ is a bounded lattice with ordering $\leqslant_{L}$ and 0 and 1 as its smallest and greatest element, respectively;

(RL.2) $(L, *, 1)$ is a commutative semigroup with neutral element 1 , i.e. $*$ is a commutative, associative $L^{2} \rightarrow L$ mapping such that $x * 1=x$, for all $x$ in $L$; 
(RL.3) $\Rightarrow$ is an $L^{2} \rightarrow L$ mapping such that, for all $x, y, z$ in $L$ :

$$
(x * y) \leqslant{ }_{L} z \text { if and only if } y \leqslant{ }_{L}(x \Rightarrow z) .
$$

It is an MTL-algebra if furthermore it satisfies, for all $x, y$ in $L$ :

(MTL.1) $(x \Rightarrow y) \vee(y \Rightarrow x)=1 \quad$ (prelinearity),

a BL-algebra if it is an MTL-algebra and for all $x, y$ in $L$ :

(BL.1) $x \wedge y=x *(x \Rightarrow y) \quad$ (divisibility),

and an MV-algebra if it is a BL-algebra and for all $x$ in $L$ :

(MV.1) $((x \Rightarrow 0) \Rightarrow 0)=x \quad$ (involutivity).

Definition 3. An algebraic structure $\mathcal{L}=(L, \wedge, \vee, \Rightarrow, 0)$ is called a Heyting algebra if, for all $a, b, c$ in $L$,

(H1) $a \Rightarrow a=b \Rightarrow b$,

(H2) $(a \Rightarrow b) \wedge b=b$,

(H3) $a \Rightarrow(b \wedge c)=(a \Rightarrow b) \wedge(a \Rightarrow c)$,

(H4) $a \wedge(a \Rightarrow b)=a \wedge b$,

(H5) $(a \vee b) \Rightarrow c=(a \Rightarrow c) \wedge(b \Rightarrow c)$,

(H6) $0 \wedge a=0$.

Now we discuss the existence of these structures on the lattice $\mathcal{L}^{I}$. Let $\mathcal{T}$ be a t-norm on $\mathcal{L}$. We define the residuum $\mathcal{I}_{\mathcal{T}}$ of $\mathcal{T}$ by, for all $x, y$ in $L^{I}$,

$$
\mathcal{I}_{\mathcal{T}}(x, y)=\sup \left\{z \mid z \in L^{I} \text { and } \mathcal{T}(x, z) \leqslant L^{I} y\right\} .
$$

If $\mathcal{T}$ satisfies the residuation principle, i.e. (RL.3) holds for $*=\mathcal{T}$ and $\Rightarrow=\mathcal{I}_{\mathcal{T}}$, then $\left(L^{I}, \wedge, \vee, \mathcal{T}, \mathcal{I}_{\mathcal{T}}, 0_{\mathcal{L}^{I}}, 1_{\mathcal{L}^{I}}\right)$ is a (distributive) residuated lattice, where $0_{\mathcal{L}^{I}}=[0,0]$ and $1_{\mathcal{L}^{I}}=[1,1]$. In [5] it is shown that such t-norms on $\mathcal{L}^{I}$ exist, ${ }^{1}$ so a residuated lattice can be built on top of $\mathcal{L}^{I}$. As a consequence, for such a t-norm, the associated intervalvalued fuzzy logic is a particular type of monoidal logic in the sense of Höhle [13], in which e.g. modus ponens holds.

On the other hand, the failure of the prelinearity condition rules out the existence of MTL-algebras on $\mathcal{L}^{I}$. To see this, assume that such a t-norm $\mathcal{T}$ exists. Let $x, y$ in $L^{I}$, then from (RL.3) it follows that

$$
x=\mathcal{T}\left(x, 1_{\mathcal{L}^{I}}\right) \leqslant{ }_{L^{I}} y \quad \text { if and only if } 1_{\mathcal{L}^{I}} \leqslant{ }_{L^{I}} \mathcal{I}_{\mathcal{T}}(x, y) .
$$

Since, for all $a=\left[a_{1}, a_{2}\right], b=\left[b_{1}, b_{2}\right]$ in $L^{I}$, it holds that $\sup (a, b)=\left[\max \left(a_{1}, b_{1}\right), \max \left(a_{2}, b_{2}\right)\right]=1_{\mathcal{L}^{I}}$ implies that $a=1_{\mathcal{L}^{I}}$ or $b=1_{\mathcal{L}^{I}}$, from (MTL.1) it follows that $\mathcal{I}_{\mathcal{T}}(x, y)=1_{\mathcal{L}^{I}}$ or $\mathcal{I}_{\mathcal{T}}(y, x)=1_{\mathcal{L}^{I}}$. Thus, using (3), we obtain that $x \leqslant_{L^{I}} y$ or $y \leqslant_{L^{I}} x$, which is a contradiction, since in $\mathcal{L}^{I}$ incomparable elements exist. Hence, the following theorem holds:

Theorem 4. There does not exist a t-norm $\mathcal{T}$ on $\mathcal{L}^{I}$ such that $\left(L^{I}, \wedge, \vee, \mathcal{T}, \mathcal{I}_{\mathcal{T}}, 0_{\mathcal{L}^{I}}, 1_{\mathcal{L}^{I}}\right)$ is an MTL-algebra.

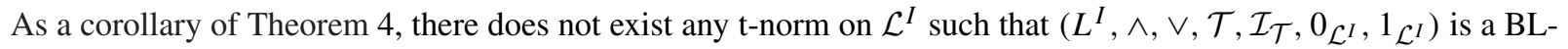
or an MV-algebra. These negative results might raise the impression that interval-valued fuzzy logics are rather weak logical systems; in what follows we argue that the bias is largely due to the importance attached to (MTL.1), and that most of the interesting properties valid in MV-algebras can be preserved by a deliberate choice of connectives.

On the unit interval it holds (see e.g. [16]) that ([0,1], min, $\left.\max , T, I_{T}, 0,1\right)$ is an MV-algebra if and only if $T$ is isomorphic to the Łukasiewicz t-norm $T_{W}$ defined by $T_{W}(x, y)=\max (0, x+y-1)$ for $x, y$ in $[0,1]$, or equivalently,

\footnotetext{
${ }^{1}$ Moreover, unlike on [0,1], on $\mathcal{L}^{I}$ condition (RL.3) is not equivalent to the left-continuity of $\mathcal{T}$; refer to [5] for an example of a continuous t-norm on $\mathcal{L}^{I}$ which does not satisfy the residuation principle.
} 
if $I_{T}$ satisfies all Smets-Magrez axioms, i.e. for all $x, y, z$ in $[0,1]$,

$$
\begin{aligned}
& I_{T}\left(I_{T}(y, 0), I_{T}(x, 0)\right)=I_{T}(x, y) \quad \text { (contrapositivity), } \\
& I_{T}\left(x, I_{T}(y, z)\right)=I_{T}\left(y, I_{T}(x, z)\right) \quad \text { (exchange principle), } \\
& I_{T}(x, y)=1 \quad \text { if and only if } x \leqslant y \quad \text { (confinement principle), } \\
& I_{T} \text { is a continuous }[0,1]^{2} \rightarrow[0,1] \text { mapping (continuity). }
\end{aligned}
$$

Clearly, this equivalence is not transferred to $\mathcal{L}^{I}$, since there does not exist an MV-algebra on $\mathcal{L}^{I}$. Nevertheless, as the following example shows, a t-norm can be constructed on $\mathcal{L}^{I}$ such that its residuum satisfies all Smets-Magrez axioms.

Example 5. In [5] the following t-norms on $\mathcal{L}^{I}$ are introduced: for $x, y$ in $L^{I}$,

$$
\begin{aligned}
& \mathcal{T}_{w}(x, y)=\left[\max \left(0, x_{1}+y_{1}-1\right), \max \left(0, x_{2}+y_{2}-1\right)\right], \\
& \mathcal{T}_{W}(x, y)=\left[\max \left(0, x_{1}+y_{1}-1\right), \max \left(0, x_{1}+y_{2}-1, x_{2}+y_{1}-1\right)\right] .
\end{aligned}
$$

Their residuum is given by, for $x, y$ in $L^{I}$,

$$
\begin{aligned}
& \mathcal{I}_{\mathcal{T}_{w}}(x, y)=\left[\min \left(1, y_{1}+1-x_{1}, y_{2}+1-x_{2}\right), \min \left(1, y_{2}+1-x_{2}\right)\right], \\
& \mathcal{I}_{\mathcal{T}_{W}}(x, y)=\left[\min \left(1, y_{1}+1-x_{1}, y_{2}+1-x_{2}\right), \min \left(1, y_{2}+1-x_{1}\right)\right] .
\end{aligned}
$$

Both these t-norms are extensions of $T_{W}$ (recall that $I_{T_{W}}$, the residuum of $T_{W}$, is given by $I_{T_{W}}(x, y)=\min (1,1-x+y)$, for all $x, y$ in $[0,1])$; the former is t-representable while the latter is not (since its second component depends also on $x_{1}$ and $\left.y_{1}\right)$.

Both $\mathcal{T}_{w}$ and $\mathcal{T}_{W}$ satisfy the residuation principle, hence $\left(L^{I}, \wedge, \vee, \mathcal{T}_{w}, \mathcal{I}_{\mathcal{T}_{w}}, 0_{\mathcal{L}^{I}}, 1_{\mathcal{L}^{I}}\right)$ and $\left(L^{I}, \wedge, \vee, \mathcal{T}_{W}, \mathcal{I}_{\mathcal{T}_{W}}\right.$, $\left.0_{\mathcal{L}^{I}}, 1_{\mathcal{L}^{I}}\right)$ are residuated lattices. In [3] it is proven that $\mathcal{I}_{\mathcal{T}_{W}}$ satisfies all Smets-Magrez axioms; on the other hand, $\mathcal{I}_{\mathcal{T}_{w}}$ is not contrapositive. So, the property that $I_{T_{W}}$ satisfies all Smets-Magrez axioms, is not inherited by the componentwise extension $\mathcal{T}_{w}$ of $T_{W}$, but by the non t-representable $\mathcal{T}_{W}$. More generally, in [3] it was proven that an $\left(L^{I}\right)^{2} \rightarrow L^{I}$ mapping $\mathcal{I}$ on $\mathcal{L}^{I}$ satisfies the Smets-Magrez axioms if and only if it is isomorphic to $\mathcal{I}_{\mathcal{T}_{W}}$.

Furthermore, using the arithmetical operators $\oplus_{\mathcal{L}^{I}}$ and $\ominus_{\mathcal{L}^{I}}$, defined in [4] by, for all $x, y$ in $\left\{\left[x_{1}, x_{2}\right] \mid\left(x_{1}, x_{2}\right) \in \mathbb{R}^{2}\right.$ and $\left.x_{1} \leqslant x_{2}\right\}$,

$$
\begin{aligned}
& x \oplus_{\mathcal{L}^{I}} y=\left[\min \left(x_{1}+y_{2}, x_{2}+y_{1}\right), x_{2}+y_{2}\right], \\
& x \ominus_{\mathcal{L}^{I}} y=\left[x_{1}-y_{2}, \max \left(x_{1}-y_{1}, x_{2}-y_{2}\right)\right],
\end{aligned}
$$

we can write $\mathcal{T}_{W}$ and its residuum in a similar way as their counterparts on the unit interval: for all $x, y$ in $L^{I}$,

$$
\begin{aligned}
& \mathcal{T}_{W}(x, y)=\sup \left(0_{\mathcal{L}^{I}}, x \ominus_{\mathcal{L}^{I}}\left(1_{\mathcal{L}^{I}} \ominus_{\mathcal{L}^{I}} y\right)\right)=\sup \left(0_{\mathcal{L}^{I}}, y \ominus_{\mathcal{L}^{I}}\left(1_{\mathcal{L}^{I}} \ominus_{\mathcal{L}^{I}} x\right)\right), \\
& \mathcal{I}_{\mathcal{T}_{W}}(x, y)=\inf \left(1_{\mathcal{L}^{I}}, y \oplus_{\mathcal{L}^{I}}\left(1_{\mathcal{L}^{I}} \ominus_{\mathcal{L}^{I}} x\right)\right) .
\end{aligned}
$$

Hence, even though the entire structure of an MV-algebra is not preserved, many important properties that are valid for the Łukasiewicz t-norm $T_{W}$ also hold for its non t-representable extension $\mathcal{T}_{W}$ (but not for the t-representable extension $\mathcal{T}_{w}$; more properties that are inherited by $\mathcal{T}_{W}$ are discussed in [5,3]).

Furthermore, the following example shows that, similarly to the unit interval, a Heyting algebra can be constructed on $\mathcal{L}^{I}$ (see also [2]).

Example 6. It can be verified that the residuum $\mathcal{I}_{\wedge}$ of $\wedge$, given by, for all $x, y$ in $L^{I}$,

$$
\mathcal{I}_{\wedge}(x, y)= \begin{cases}1_{\mathcal{L}^{I}} & \text { if } x \leqslant L^{I} y, \\ {\left[y_{2}, y_{2}\right]} & \text { if } x_{1} \leqslant y_{1} \text { and } x_{2}>y_{2}, \\ {\left[y_{1}, 1\right]} & \text { if } x_{1}>y_{1} \text { and } x_{2} \leqslant y_{2}, \\ y & \text { else, }\end{cases}
$$

satisfies (H.1)-(H.6), so $\left(L^{I}, \wedge, \vee, \mathcal{I}_{\wedge}, 0_{\mathcal{L}^{I}}\right)$ is a Heyting algebra.

Finally, note that in $\left(L^{I}, \wedge, \vee, \wedge, \mathcal{I}_{\wedge}, 0_{\mathcal{L}^{I}}, 1_{\mathcal{L}^{I}}\right)$, the divisibility condition (BL.1) holds, but not the involutivity condition (MV.1); in $\left(L^{I}, \wedge, \vee, \mathcal{T}_{W}, \mathcal{I}_{\mathcal{T}_{W}}, 0_{\mathcal{L}^{I}}, 1_{\mathcal{L}^{I}}\right)$ on the other hand, (MV.1) is valid but not (BL.1); in general, in a 
residuated lattice $\left(L^{I}, \wedge, \vee, \mathcal{T}, \mathcal{I}_{\mathcal{T}}, 0_{\mathcal{L}^{I}}, 1_{\mathcal{L}^{I}}\right),($ BL.1) and (MV.1) cannot hold simultaneously, due to a result of Höhle [13] stating that (BL.1) and (MV.1) taken together imply (MTL.1).

\section{Conclusion-the challenges ahead}

While the study of IVFSs and their intended semantics has received ample attention, and their application in knowledge-based systems is widely understood and promoted, the research on interval-valued fuzzy logic in the narrow sense is still at the very outset.

Up to now the research on fuzzy logic in the narrow sense mainly focussed on prelinear structures, such as MTL-, BL- and MV-algebras (which are all generalizations of structures generated by special classes of t-norms on [0, 1]). However, from our exposition, it is clear that the underlying lattice $\mathcal{L}^{I}$ of interval-valued fuzzy logic does not fit in that framework, due to the failure of the prelinearity condition; we can only assert at this moment that residuated lattices (and Heyting algebras) can be constructed on top of $\mathcal{L}^{I}$.

Therefore, an important issue that has to be dealt with in the future, is whether suitable residuated structures can be found in which prelinearity and divisibility are not necessarily satisfied but in which a maximal number of properties valid in MTL- and other algebras do hold. Similarly as for the prelinear structures, the new structures should form generalizations of the structures generated by special classes of t-norms on $\mathcal{L}^{I}$ that satisfy the residuation principle. The $\mathrm{t}$-norm $\mathcal{T}_{W}$, which has a behaviour that is surprisingly similar to the Łukasiewicz t-norm on [0, 1], acts as an elegant role model for what can be achieved with interval-valued logic, and in particular with non t-representable connectives. So, what we propose is to extend the well-studied prelinear structures such as MTL-, BL- and MV-algebras to more general structures in which prelinearity and divisibility do not necessary hold and which, when considered in the framework of IVFSs, are closely related to some special classes of t-norms on $\mathcal{L}^{I}$.

Another challenge concerns completeness of the generated logics: e.g. given a class $\mathbb{\mathbb { T }}$ of t-norms on $\mathcal{L}^{I}$, find a set of axioms and deduction rules, and the most general class $\mathbb{K}$ of residuated lattices such that

- each formula that can be proven from the axioms using one or more deduction rules, is a tautology, i.e. evaluates to $1_{\mathcal{L}^{I}}$ whatever the value of the propositional variables, for each $\mathcal{L}$ in $\mathbb{K}$;

- each formula that is a tautology for each $\mathcal{L}$ in $\mathbb{R}$ is provable;

- each formula that is a tautology for each $\left(L^{I}, \wedge, \vee, \mathcal{T}, \mathcal{I}_{\mathcal{T}}, 0_{\mathcal{L}^{I}}, 1_{\mathcal{L}^{I}}\right)$, where $\mathcal{T}$ is a t-norm from $\mathbb{T}$, is also a tautology for each $\mathcal{L}$ in $\mathbb{K}$.

In conclusion, we envisage that the formalization of interval-valued fuzzy logics with Łukasiewicz-like properties will generate an interesting parallel development to the mainstream of prelinear fuzzy logics.

\section{References}

[1] K.T. Atanassov, Intuitionistic fuzzy sets VII ITKR's Session, Sofia (deposed in Central Sci.-Technical Library of Bulg. Acad. of Sci. 1697/84) (in Bulgarian) (1983).

[2] G. Cattaneo, D. Ciucci, Intuitionistic fuzzy sets or orthopair fuzzy sets?, in: M. Wagenknecht, R. Hampel (Eds.), Proceedings of Third International Conference on Fuzzy Logic and Technology, 2003, pp. 159-163.

[3] C. Cornelis, G. Deschrijver, E.E. Kerre, Implication in intuitionistic and interval-valued fuzzy set theory: construction, application, Internat. J. Approx. Reason. 35 (1) (2004) 55-95.

[4] G. Deschrijver, Algebraic operators on $\mathscr{L}^{I}$, submitted for publication.

[5] G. Deschrijver, C. Cornelis, E.E. Kerre, On the representation of intuitionistic fuzzy t-norms and t-conorms, IEEE Trans. Fuzzy Systems 12 (1) (2004) 45-61.

[6] F. Esteva, L. Godo, Monoidal t-norm based logic: towards a logic for left-continuous t-norms, Fuzzy Sets and Systems 114 (3) (2001) 271-288.

[7] W.L. Gau, D.J. Buehrer, Vague sets, IEEE Trans. Systems, Man Cybernetics 23 (2) (1993) 610-614.

[8] M. Gehrke, C. Walker, E. Walker, Some comments on interval-valued fuzzy sets, Internat. J. Intelligent Systems 11 (1996) 751-759.

[9] J. Goguen, L-fuzzy sets, J. Math. Anal. Appl. 18 (1967) 145-174.

[10] M.B. Gorzałczany, A method of inference in approximate reasoning based on interval-valued fuzzy sets, Fuzzy Sets and Systems 21 (1) (1987) $1-17$.

[11] I. Grattan-Guinness, Fuzzy membership mapped onto interval and many-valued quantities, Z. Math. Logik Grundlag. Mathe. 22 (1975) 149-160.

[12] P. Hájek, Metamathematics of Fuzzy Logic, Kluwer Academic Publishers, Dordrecht, 1998.

[13] U. Höhle, Commutative residuated l-monoids, in: U. Höhle, E.P. Klement (Eds.), Non-Classical Logics and Their Applications to Fuzzy Subsets, Kluwer Academic Publishers, Dordrecht, 1995, pp. 53-106. 
[14] K.U. Jahn, Intervall-wertige Mengen, Math. Nach. 68 (1975) 115-132.

[15] S. Jenei, A more efficient method for defining fuzzy connectives, Fuzzy Sets and Systems 90 (1) (1997) 25-35.

[16] E.P. Klement, R. Mesiar, E. Pap, Triangular Norms, Kluwer Academic Publishers, Dordrecht, 2002.

[17] J.M. Mendel, Uncertain Rule-based Fuzzy Logic Systems, Prentice-Hall PTR, Upper Saddle River, NJ, 2001.

[18] V. Novák, I. Perfilieva, J. Močkoř, Mathematical Principles of Fuzzy Logic, Kluwer Academic Publishers, Dordrecht, 1999.

[19] R. Sambuc, Fonctions $\Phi$-floues. Application à l'aide au diagnostic en pathologie thyroidienne, Ph. D. Thesis, University of Marseille, 1975.

[20] I.B. Türkşen, Interval valued fuzzy sets based on normal forms, Fuzzy Sets and Systems 20 (2) (1986) 191-210.

[21] L. Zadeh, The concept of a linguistic variable and its application to approximate reasoning-I, Inform. Sci. 8 (1975) 199-249. 